\title{
PROGNOSTIC CRITERIA FOR POST-OPERATIVE SUCCESS IN PATIENTS UNDERGOING SURGERY FOR ADULT SPINAL DEFORMITIES
}

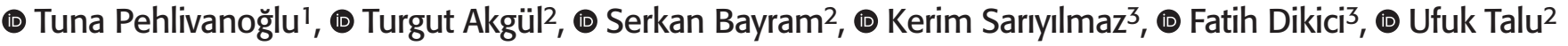 \\ ${ }^{1}$ Emsey Hospital, Department of Orthopedic Surgery and Traumatology, Advanced Spine Surgery Center; Yeni Yüzyıl University, Faculty of \\ Health Sciences, Istanbul, Turkey \\ 2istanbul University, Istanbul Faculty of Medicine, Department of Orthopedic Surgery and Traumatology, İstanbul, Turkey \\ ${ }^{3}$ Acıbadem University Faculty of Medicine, Department of Orthopedic Surgery and Traumatology, Istanbul Turkey
}

\begin{abstract}
Objective: The current reported rate of revision after surgery for adult spinal deformities (ASDs) is up to $45 \%$. The aim of this study was to analyze patients with ASD who underwent failed primary surgery and required revision surgeries, in order to identify the reasons for failure and revision, while assessing possible prognostic criteria.

Materials and Methods: Thirty-two patients ( 27 women, 5 men) with a mean age of 69.8 years and follow-up period of 44.6 months were included. Before first revision, patients had a mean sagittal vertical axis (SVA) of $94.2 \mathrm{~mm}$, lumbar lordosis (LL) of $33.3^{\circ}$, thoracic kyphosis (TK) of $35.3^{\circ}$, pelvic incidence (PI) of $56.9^{\circ}$, pelvic tilt (PT) of $27.8^{\circ}$, PI-LL of $24.9^{\circ}$, and coronal Cobb angle of $22^{\circ}$. Mean duration from the initial surgery until the first revision was 34.8 months. Fusion levels extended from T1 to S2. Twenty patients received transforaminal lumbar interbody fusion and 9 received anterior lumbar interbody fusion cages. Five patients underwent corpectomy combined with anterior cage. Three patients underwent ponte-, and 7 underwent pedicle subtraction osteotomies.

Results: After the last revision surgery, patients' sagittal plane parameters were significantly corrected ( $p<0.001$ for mean SVA, LL, PT, PI-LL mismatch and coronal Cobb). The most frequent reason for revision was found as advanced sagittal malalignment (ASM) in 29 patients (90.6\%) followed by proximal junctional kyphosis (PJK) in 13 patients (40.6\%). The most common surgical planning mistakes leading to revision were detected as proximal short fusion extending to thoracolumbar junction and not to T10, thus avoiding the stabilizing effect of the rib cage in 18 patients (56.3\%); followed by no bone cement [polymethylmethacrylate (PMMA)] augmented screw application despite documented osteoporosis in 17 patients (53.1\%).

Conclusion: The present study concluded that ASM and PJK were the most common reasons for revision following ASD surgery, while short proximal level of instrumentation and not placing PMMA augmented pedicle screws in patients with documented osteoporosis were the most common surgical planning pitfalls leading to revision.

Keywords: Adult spinal deformity, spinal deformity correction surgery, revision surgery, causes of failure, prognostic criteria, pitfalls of surgical planning
\end{abstract}

\section{Introduction}

Adult spinal deformity (ASD), which might be the result of the developmental deformities at younger ages or might occur de novo in older ages as a result of degenerative changes in addition to tumors, infections or traumas as well as to a wide range of other etiologies, constitute a serious public health problem especially for geriatric population by causing physical limitations leading to remarkably diminished quality of life ${ }^{(1-3)}$. With the increase of life expectancy, the ideal treatment of ASD was shifted towards surgical options, especially for patients over the age of 60 , and in cases accompanied by the progression of spinal deformity and neurological compression resulting in intractable pain and severe physical limitations yielding a remarkable decline in quality of life ${ }^{(4,5)}$. Hence, the often extensive surgical treatment for ASD was aimed to provide clinical amelioration by reducing the pain together with the deformity and allowing the patients to be freed from physical restrictions providing an improved quality of life ${ }^{(6,7)}$.

Surgeries for ASD were reported to be prone to risks including neurologic and cardiovascular complications ${ }^{(8)}$. Despite modern instrumentation techniques, rates of revision surgeries after primary ASD surgeries were detected to be on the rise, ranging from $9 \%$ to $45 \%$, with implant failure, pseudoarthrosis and 
turkishspine

adjacent segment disease and infection has been reported frequently as common reasons ${ }^{(9,10)}$.

Revision ASD surgery is a more technically demanding procedure as compared to primary surgery for ASD with 20 to $50 \%$ rates of complications ${ }^{(11,12)}$. The aim of this study was to analyze the potential risk factors of patients who underwent failed surgery due to ASDs and required revision surgeries, and to assess possible pitfalls regarding surgical planning, which might be associated with a potential revision surgery, in addition to the evaluation of the sagittal parameters, clinical and functional outcomes together with the health related quality of life of patients revised after failed primary surgery for ASD.

\section{Materials and Methods}

After obtaining institutional review board approval (İstanbul University, İstanbul Faculty of Medicine, Department of Orthopedic Surgery and Traumatology, Nr: 2019/068), a retrospective analysis was undertaken to detect a consecutive group of patients, who had a primary diagnosis of ASD and was operated in a single institution between 2010-2015. Two hundred and eighteen consecutive patients were detected to be operated in the aforementioned time interval in a single institution for the management of ASD.

Patients were enrolled in the present study on the basis of the following inclusion criteria: (1) Having a diagnosis of primary ASD; (2) being skeletally mature ( $>18$ years of age); (3) having been revised after the primary operation for ASD for following reasons: Advanced sagittal imbalance, proximal junctional kyphosis (PJK), pseudoarthrosis, implant failure, infection, neurologic deficit; (4) having a minimum follow-up duration of 2 years; (3).

Exclusion criteria comprised: (1) Having a diagnosis other than primary ASD; (2) being skeletally immature (<18 years of age); (3) having only primary surgery for ASD with no revision; (4) having a minimum follow-up duration of less than 2 years; (5) having a diagnosis of acute trauma or tumor; (6) incomplete radiographic documentation; (7) being unwilling to participate in the study (Table 1).

As a result of the exclusion criteria 186 patients (143: Having only primary surgery for ASD with no revision; 18: Having a diagnosis of acute trauma-tumor; 11: Having a diagnosis other than primary ASD; 8: Incomplete radiographic documentation; 6: Unwilling to participate) were excluded from the study. The remaining 32 patients ( 27 women, 5 men) were included in the study (Table 2).

\section{Radiographic Outcome Parameters (ROP)}

Pre-operative and post-operative radiographic measurements were undertaken on standing whole spine posteroanterior and lateral X-rays. Computer tomography scans were utilized to evaluate implant placements and the stage of bony union; while magnetic resonance imaging was used to evaluate the current status of neural structures as well as the posterior ligamentous structures, if necessary and available.
The radiographic measurements regarding the sagittal parameters including sagittal vertical axis (SVA), pelvic incidence (PI), pelvic tilt (PT), thoracic kyphosis (TK), lumbar lordosis (LL) and PI-LL mismatch together with coronal Cobb angle of the de novo scoliotic curves were undertaken by one independent senior spine surgeon with Surgimap software (Nemaris Inc., New York, NY, USA). X-Rays were taken pre-operatively, immediate postoperatively, at the $1^{\text {st }}$ (first outpatient visit), $3^{\text {rd }}$ and $6^{\text {th }}$ month, annually and at the latest FU appointment.

\section{Clinical Outcome Parameters}

As patient reported outcome questionnaires including visual analogue scale (VAS) score for pain and oswestry disability index (ODI) scores were applied to evaluate the clinical and functional outcomes pre-operatively and at the latest followup appointment. To assess the quality of life, SF-36 scores [mental component score (MCS) and physical component score (PCS)] were utilized.

\section{Post-operative Rehabilitation Protocol}

Patients were mobilized immediately after surgery and were allowed to return to daily activities after discharge, while return to sportive activities (including non-contact sports, swimming and light gym) were allowed after $6^{\text {th }}$ post-operative month if they required to exercise.

\section{Information of Informed Consent}

All patients were taken informed consents, so that their pre-, intra- and post-operative data including the X-rays could be used for publication by hiding their identity.

\section{Statistical Analysis}

For the statistical analysis, SPSS software (Version 22.0; SPSS Inc, Chicago, IL, USA) was used. Data are expressed as mean +/- SD (standard deviation). The chi-square test and Fisher's exact test were used for the analysis of categorical variables and to compare different time points where appropriate. OneWay analysis of variance (ANOVA) was used to determine a significant difference at various time points. A p-value less than 0.05 was considered as statistically significant.

\section{Results}

Thirty-two patients (27 females, 5 males) with a mean age of 69.8 (range 60-84) and mean follow-up duration of 44.6 months (range 24-120) were included Table 3. Thirty-one patients were confirmed to have osteoporosis by using bone densitometry (96.9\%).

Patients had primary diagnoses before the index surgery for ASD as: Isolated lumbar degenerative spondylolisthesis and sagittal malalignment in 2 patients (6.3\%); degenerative spondylolisthesis and sagittal malalignment accompanied by neural compression due to disc herniation and/or spinal stenosis in 12 patients (37.5\%); sagittal imbalance due to osteoporotic 
vertebrae fractures (4 isolated lumbar, 1 lumbosacral junction, 1 multiple thoracic and lumbar) in 6 patients (18.7\%); isolated coronal imbalance due to de novo scoliosis accompanied by lumbar disc herniation in 3 patients (9.4\%); coronal imbalance due to combined sagittal imbalance and de novo scoliosis accompanied by lumbar disc herniation and/or spinal stenosis in 9 patients (28.1\%) Table 3.

The rate of revision in the present study population was calculated as $14.7 \%$. Patients had a mean time from the primary surgery to the first revision as 34.8 months (range 6-120), while 6 patients were revised more than 1 time (18.8\%) (Table 3 ).

Main reasons for revision were detected as: Advanced sagittal imbalance in 29 patients (90.6\%); PJK in 13 patients (40.6\%); pseudoarthrosis (L5-S1:7, L4-L5:2, L3-L4:2) in 11 patients (34.4\%); coronal imbalance due to de novo scoliosis in 8 patients (25\%); implant failure (screw pull-out) in 7 patients (21.9\%). In some patients, more than more than one pre-revision diagnosis was present at the same time (Table 4).

Main mistakes regarding the surgical planning leading to revision were detected as: Short proximal fusion extending to thoracolumbar junction and not to T10, thus avoiding the stabilizing effect of the rib cage in 18 patients (56.3\%); no bone cement polymethyl methacrylate (PMMA) augmented screw application despite documented osteoporosis in 17 patients (53.1\%); continuing sagittal imbalance as a result of the failed primary surgery and/or failed previous revision surgery in 15 patients (46.9\%); avoiding to perform transforaminal lumbar interbody fusion (with cage) despite the instrumentation of L5-S1 in 7 patients (21.9\%); problems regarding the design of the constructs leading to biomechanical failure in 5 patients (15.6\%); application of kyphoplasty only without further correction of sagittal imbalance in 2 patients (6.3\%). In some patients, more than one pre-revision surgical planning mistake was present at the same time (Table 4).

Revision procedures together with types of osteotomies, applied to the study population, were summarized in Table 5, Figure 1.
Patients' pre-operative mean VAS-back score of 7.4 (range 6-8) and VAS-leg score of 6.1 (range 5-7) were improved to 2.6 (range 2-4) and 2.8 (range 1-4), respectively at latest follow-up with high statistical significance ( $<0.001$ for both). Pre-operative ODI scores of 68.4 (range 61-73) were detected to be improved to 21.3 (range 17-28) at latest follow-up with high statistical significance as well $(p<0.001)$. Pre-operative SF-36 MCS of 46.2 (range 45.8-49.7) and PCS of 45.1 (42.6-48.3) indicating health related quality of life were detected to be improved to 54.8 (range 52.6-57.2) and 55.7 (54.3-57.2) respectively at the latest follow-up with high statistical significance ( $p<0.001$ for both) (Table 6).

Sagittal parameters including SVA, PT and PI-LL mismatches were detected to be improved with high statistical significance at the last follow-up ( $p<0.001$ for all), together with the remarkable improvement of $L L(p<0.001)$, while the TK and PI at the last follow-up remained similar to pre-operative values ( $p>0.05$ ) (Table 7).

No intraoperative complications were acquired. All patients were neurologically intact early post-op and at the latest follow-up visit. Three patients at developed radicular pain with

Table 2. Flowchart of the study population

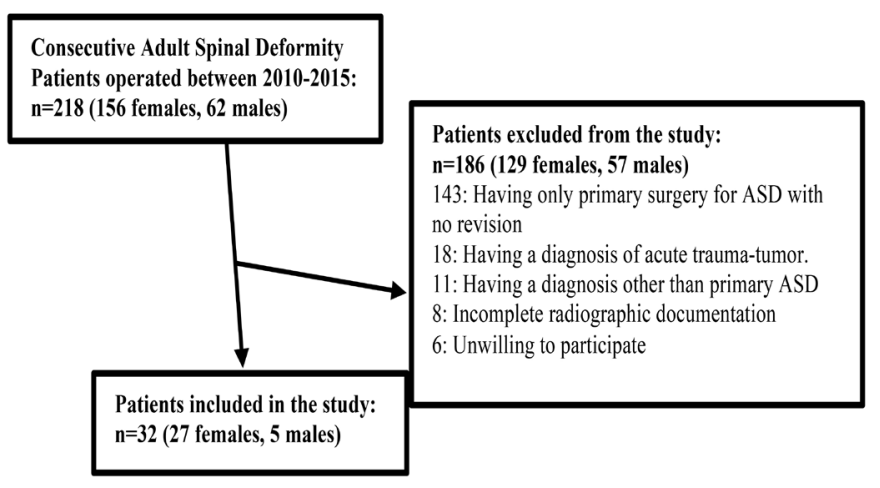

Table 1. Inclusion and exclusion criteria

\section{Inclusion Criteria}

Having a diagnosis of primary adult spinal deformity (ASD)

Being skeletally mature ( $>18$ years of age)

\section{Exclusion Criteria}

Having a diagnosis other than primary adult spinal deformity (ASD)

Being skeletally immature ( $<18$ years of age)

Having been revised after the primary operation for ASD for following reasons:

-Advanced sagittal imbalance

-Proximal junctional kyphosis (PJK)

-Pseudoarthrosis

-Implant failure

-Infection

-Neurological deficit

Having a minimum follow-up duration of 2 years
Having a follow-up duration of less than 2 years

Having a diagnosis of acute trauma or tumor Incomplete radiolographic documentation

Being unwilling to participate in the study 
no motor deficit early postoperative and were managed with conservative therapy. Two patients early post-operatively had superficial wound problems resolved with local wound care and appropriate dressings. No major complications including implant failure or pseudoarthrosis were encountered at the latest follow-up visit.

Table 3. Data regarding the patients' characteristics

\begin{tabular}{|ll|}
\hline Number of patients & 32 (27 females, 5 males) \\
\hline Mean age of patients & 69.8 (range 60-84) \\
\hline Average duration of follow-up & 44.6 (range 24-120) \\
\hline
\end{tabular}

12 Patients: degenerative spondylolisthesis and sagittal malalignment accompanied by neural compression due to disc herniation and/or spinal stenosis

9 Patients: Coronal imbalance due to combined sagittal imbalance and de novo

Primary diagnoses of patients before the scoliosis accompanied by lumbar disc herniation and/or spinal stenosis

6 Patients: sagittal imbalance due to osteoporotic vertebrae fractures (4 isolated lumbar, 1 lumbosacral junction, 1 multiple thoracic and lumbar)

3 Patients: Isolated coronal imbalance due to de novo scoliosis accompanied by lumbar disc herniation.

2 Patients: Isolated lumbar degenerative spondylolisthesis and sagittal malalignment

Rate of revision

Mean time from the primary surgery to the first revision

Patients revised more than one time
$14.7 \%$

34.8 months (range 6-120)

$6(18.8 \%)$

Table 4. Reasons for revision-surgical planning mistakes leading to revision

29 Patients (90.6\%): Advanced sagittal imbalance

13 Patients (40.6\%): Proximal junctional kyphosis (PJK)

Main reasons for revision

11 Patients (34.4\%): Pseudoarthrosis (L5-S1:7, L4-L5:2, L3-L4:2)

8 Patients (25\%): Coronal imbalance due to de novo scoliosis

7 Patients (21.9\%): Implant failure (screw pull-out)

18 Patients (56.3\%): Short proximal fusion extending to thoracolumbar junction and not to T10, thus avoiding the stabilizing effect of the rib cage

17 Patients (53.1\%): No bone cement (PMMA) augmented screw application despite documented osteoporosis

Main mistakes regarding surgical 15 Patients (46.9\%): Continuing sagittal imbalance as a result of the failed primary surgery and/ or failed previous revision surgery

planning leading to revision

7 Patients (21.9\%): Avoiding to perform TLIF (with cage) despite the instrumentation of L5-S1

5 Patients (15.6\%): Problems regarding the design of the constructs leading to biomechanical failure

2 Patients (6.3\%): Application of kyphoplasty only without further correction of sagittal imbalance in 2 patients

TLIF: Transforaminal lumbar interbody fusion, PMMA: Polymethyl methacrylate

Table 5. Revision procedures

\begin{tabular}{|ll|}
\hline Number of patients & Revision procedure \\
\hline $32(100 \%)$ & Elongation of posterior fusion levels (between T1-S2) \\
\hline $12(37.5 \%)$ & Extension of distal fusion level to S2 and performing spinopelvic fixation by using S2-iliac-alar screws \\
\hline $20(62.5 \%)$ & Posterior interbody fusion by using TLIF with autograft filled cages (between L1-S1 at various levels) \\
\hline $9(28.1 \%)$ & Anterior interbody fusion by using ALIF with autograft filled cages (at L5-S1 only) \\
\hline $5(15.6 \%)$ & $\begin{array}{l}\text { Total -/ hemicorpectomy + insertion of an autograft filled expandable cage (2: L1 hemicorpectomy, } 1 \text { T11 } \\
\text { hemi-, T12 total, L1 hemicorpectomy, 1 L2-3 hemicorpectomy, 1 T12 hemi-, L1 total corpectomy) }\end{array}$ \\
\hline $24(75 \%)$ & Bone cement (PMMA) augmented, fenestrated pedicle screws \\
\hline $3(9.3 \%)$ & Ponte osteotomy (1 one level, 1 two levels) \\
\hline $7(21.9 \%)$ & Pedicle subtraction osteotomy (PSO) (6 L3,1 L3 and L4) \\
\hline PMMA: Polymethyl methacrylate,ALIF:Anterior lumbar interbody fusion
\end{tabular}

PMMA: Polymethyl methacrylate, ALIF: Anterior lumbar interbody fusion 


\section{Discussion}

Revision procedures following primary surgeries or previous revisions of patients with ASD could be considered as one of the most challenging procedures among all operations in context of spine surgery, because these particular operations were reported to be prone to many complications and further revisions as a result of individual patients' characteristics, comorbidities and technical problems related to prior surgeries; thus they must be individualized in order to obtain optimal

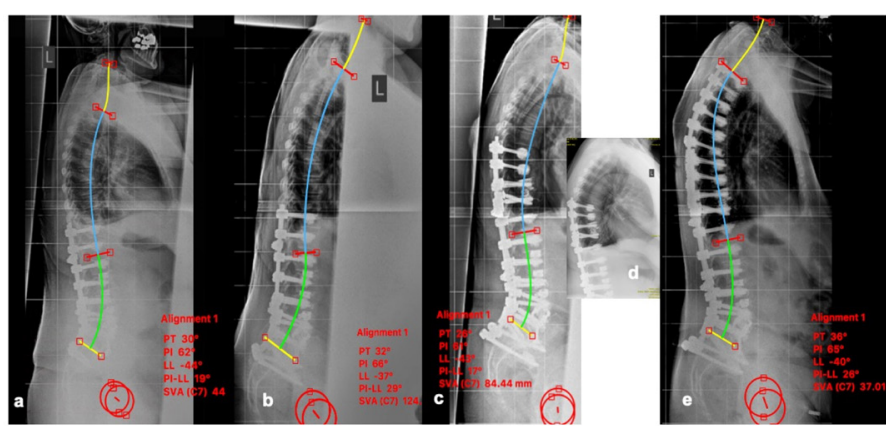

Figure 1. A 77-year old female patient primarly operated for ASD a: Standing early post-op lateral X-ray showing good global sagittal balance. $\mathbf{b}: 1^{\text {st }}$ year postoperative. Advanced positive sagittal imbalance due to implant failure. Revision planned: Extension of the instrumentation to: T8-S2. S2-alar-iliac instrumentation. Spinopelvic fixation. PMMA augmented, fenestrated pedicle screw application. c: Standing early postperative lateral X-ray showing restored global sagittal balance ( $1^{\text {st }}$ revision). d: PJK detected 6 months after the first revision. $2^{\text {nd }}$ revision planned: Proximal extension of the construct to T1.e: $1^{\text {st }}$ year after the second revision. Global sagittal balance is restored ASD: Adult spinal deformity, PMMA: Polymethyl methacrylate, PJK: Proximal junctional kyphosis

Table 6. Clinical and functional outcomes

\begin{tabular}{llll}
\hline & Pre-operative & At the last follow-up & p-value \\
\hline Mean VAS-back & 7.4 (range 6-8) & $2.6($ range $2-4)$ & $<0.001$ \\
\hline Mean VAS-leg & 6.1 (range 5-7) & $2.8($ range 1-4) & $<0.001$ \\
\hline Mean ODI score & 68.4 (range 61-73) & $21.3($ range 17-28) & $<0.001$ \\
\hline Mean SF-36 MCS & 46.2 (range 45.8-49.7) & $54.8($ range 52.6-57.2) & $<0.001$ \\
\hline Mean SF-36 PCS & 45.1 (range 42.6-48.3) & 55.7 (range 54.3-57.2) & $<0.001$ \\
\hline
\end{tabular}

VAS: Visual analogue scale, ODI: Oswestry disability index, MCS: Mental component score, PCS: Physical component score

Table 7. Radiographic outcomes

\begin{tabular}{|c|c|c|c|}
\hline & Pre-operative & At the last follow-up & p-value \\
\hline Mean SVA (mm) & 94.2 (range -14.2-226.7) & 46.5 (range 2-122.8) & $<0.001$ \\
\hline Mean coronal malalignment (mm) & 81.4 (range $47.1 /-121.9$ ) & 12.1 (range $3.5-62.1$ ) & $<0.001$ \\
\hline Mean lumbar lordosis $\left({ }^{\circ}\right)$ & 33.3 (range 3-54) & 46.2 (range $20-71$ ) & $<0.001$ \\
\hline Mean pelvic incidence $(\mathrm{PI})\left(^{\circ}\right)$ & 56.9 (range 35-82) & 57.3 (range 39-88) & 0.42 \\
\hline Mean PI-LL mismatch & 24.9 (range $1-48$ ) & 13.4 (range 0-39) & $<0.001$ \\
\hline Mean coronal cobb angle $\left({ }^{\circ}\right)$ & 22 (range 9-35) & 3 (range $2-4$ ) & $<0.001$ \\
\hline
\end{tabular}

SVA: Sagittal vertical axis results with the aim to reduce further revisions ${ }^{(3,13)}$.

The present study reported a rate of revision of $14.7 \%$, which was in conjunction with the current literature reporting a wide spectrum of rates of revision ranging from $9 \%$ to $45 \%^{(2,10)}$. Hence, that particular wide range of rates of revision procedures were attributed by previous studies to individual patients characteristics including age, comorbidities, status of osteoporosis, as well as types of previously applied procedures, length of follow-up and advancements in surgical strategies ${ }^{(1,2,9)}$. Nevertheless it is an inevitable fact, that despite the surgical advancements including the application of modern instrumentation methods and advanced methods to treat osteoporosis, and osteobilogics, rates of revisions for ASD surgery were not detected to be improving in recent years ${ }^{(11,13,14)}$. The most common reason for revision was reported as implant failure by many studies. The results of the present study were also in conjunction with the current literature, with advanced sagittal imbalance due to implant failure was reported a common reason for revision. Implant failure was reported by Poorman et al. ${ }^{(15)}(43.1 \%)$, Pitter et al. ${ }^{(2)}(38.2 \%)$ and Martini et al. ${ }^{(3)}(36 \%)$ as the most common reason for revision. The present study reported an implant failure rate of $21.9 \%$ resulted in revision surgery especially attributed to screw pull-out in the fusion construct. In addition to that, in line with the numbers in the recent literature, among patients with advanced sagittal imbalance, 12 cases with a rate of $37.5 \%$ were found out to have implant failure as well. To overcome implant failure, proximal and distal extension of the construct with various rod combinations has been advised. While Kelly et al. ${ }^{(10)}$ reported to extend the construct to ilium as standard of care in revision 
surgery, Martini et al.(3) recommended the application of deltarod, tie-rod and kickstand rod configurations. We preferred to expand the construct to S2 and to apply S2-alar-iliac screws resulting in spinopelvic fixation in conjunction with the literature.

PJK was reported by many studies as the second most common (after implant failure) reason for revision after ASD surgery ${ }^{(3,10)}$, while Kim et al. ${ }^{(16)}$ reported PJK as the most common cause of revision surgery after primary surgery for ASD. The present study, in conjunction with the majority of the current literature also reported PJK as the second most common cause of revision surgery following primary surgery for ASD. To overcome PJK, more proximal extension of the instrumentation in addition to less constrained instrumentation at the transition zone and application of prophylactic vertebroplasty to adjacent level while augmenting those levels with bone cement has been suggested and reported varying rates of success regarding the prevention of PJK ${ }^{(17-20)}$. In the present study, to prevent further PJK, proximal extension of the instrumentation while keeping it less constrained at the transition zone together with protection of the integrity of posterior ligamentous structures at the most proximal level of the construct was undertaken.

Pseudoarthrosis was reported to be one of the main causes for revision surgery after primary interventions for $\operatorname{ASD}^{(2,15)}$. Kelly et al. ${ }^{(10)}$ underlined, that pseudoartrosis was the most common reason for the index revision surgery regarding patients with ASD. A repeat surgery rate of $10 \%$ was reported by some literature to underline pseudoarthrosis, which if multiple was likely to provide subsequent pseudoarthrosis especially at transitional areas (thoracolumbar and lumbosacral junctions) necessitating multiple revision surgeries ${ }^{(21,22)}$. To overcome pseudoarthrosis, the importance of anterior and posterior interbody fusion by using interbody cages filled with autografts was underlined ${ }^{(3,14,21)}$. It was also suggested, that the usage of bone morphogenic protein might be helpful to achieve fusion with better quality ${ }^{(15,23)}$. In line with the current literature, the present study also utilized anterior and posterior interbody fusion by using autograft filled interbody cages especially at the level of L5-S1 as a routine in revisions for ASD, while L5-S1 pseudoarthrosis was also reported frequently necessitating L5S1 interbody fusion and spinopelvic fixation ${ }^{(24,25)}$.

The usage of major osteotomies by aiming optimal correction in both coronal and sagittal planes has been accepted as a current standart of care in terms of primary and revision surgeries for $\operatorname{ASD}^{(3,6,26)}$. Among the high variety of osteotomies, pedicle subtraction osteotomy (PSO) has especially been used more frequently because of the high angular correctional efficacy up to $30^{\circ}(27,28)$. In the present study, in conjunction with the literature 7 PSOs and 3 ponte osteotomies were utilized. Restoration of global sagittal alignment was reported to be of crucial importance by yielding improved clinical outcomes, while the magnitude of kyphosis -sagittal imbalance- was positively correlated with the worsening of quality of life $\mathrm{e}^{(6,13)}$. Martini et al.(3) showed a successful restoration of the global sagittal balance with improved PT and PI-LL values, correlated with the improvement of clinical and functional scores (including VAS, ODI, SF-36), as did Hu and Lieberman ${ }^{(7)}$. The present study, in conjunction with the literature reported, that restoration of sagittal balance was accompanied with significantly improved VAS led/back, ODI and SF-36 MCS/PCS scores underlining the excellent clinical-functional outcomes together with remarkable improvements regarding the health related quality of life.

\section{Study Limitations}

One of the limitations of the present study is its retrospective nature. Another limitation is the relatively limited number of patients, which is owed to the strict inclusion criteria that were defined to obtain a homogenous group of patients.

\section{Conclusion}

The present study concluded, that $14.7 \%$ of patients who had a primary surgery for ASD required one or more revision surgeries. Advanced sagittal malalignment followed by PJK were the most frequent reasons for revision following ASD surgery, in addition to short proximal level of instrumentation and not placing PMMA augmented pedicle screws in cases with documented osteoporosis, which were detected as the most frequent surgical planning pitfalls leading to revision following primary ASD surgery. It was concluded, that with individualized surgical planning, the global sagittal alignment could be restored and excellent clinical and functional scores could be obtained.

\section{Ethics}

Ethics Committee Approval: Institutional review board approval was taken from İstanbul University, İstanbul Faculty of Medicine, Department of Orthopedic Surgery and Traumatology ( $\mathrm{Nr}$ : 2019/068).

Informed Consent: A retrospective analysis was undertaken to detect a consecutive group of patients, who had a primary diagnosis of ASD.

Peer-reviewed: Internally peer-reviewed.

\section{Authorship Contributions}

Concept: T.P., Design: T.P., U.T., Data Collection or Processing: T.P., T.A., S.B., K.S., F.D., U.T., Analysis or Interpretation: T.P., U.T., Literature Search: T.P., T.A., S.B., K.S., F.D., U.T., Writing: T.P.

Conflict of Interest: The authors declare that they have no conflict of interest.

Financial Disclosure: The authors declared that this study received no financial support.

\section{REFERENCES}

1. Ames CP, Scheer JK, Lafage V, Smith JS, Bess S, Berven SH, et al Adult spinal deformity: epidemiology, health impact, evaluation, and management. Spine Deform. 2016;4:310-22.

2. Pitter $F T$, Lindberg-Larsen $M$, Pedersen $A B$, Dahl $B$, Gehrchen $M$. Revision risk after primary adult spinal deformity surgery: a nationwide study with two-year follow-up. Spine Deform. 2019;7:619-626.e2. 
3. Martini C, Langella F, Mazzucchelli L, Lamartina C. Revision strategies for failed adult spinal deformity surgery. Eur Spine J. 2020;29(Suppl 1):116-25.

4. Sing DC, Berven SH, Burch S, Metz LN. Increase in spinal deformity surgery in patients age 60 and older is not associated with increased complications. Spine J. 2017;17:627-35.

5. Smith JS, Fu K-M, Urban P, Shaffrey Cl. Neurological symptoms and deficits in adults with scoliosis who present to a surgical clinic: incidence and association with the choice of operative versus nonoperative management. J Neurosurg Spine. 2008;9:326-31.

6. Glassman SD, Bridwell K, Dimar JR, Horton W, Berven S, Schwab F. The impact of positive sagittal balance in adult spinal deformity. Spine (Phila Pa 1976). 2005;30:2024-9.

7. Hu X, Lieberman IH. Revision adult spinal deformity surgery: does the number of previous operations have a negative impact on outcome? Eur Spine J. 2019;28:155-60.

8. Smith JS, Shaffrey $\mathrm{Cl}$, Glassman SD, Berven SH, Schwab FJ, Hamill CL, et al. Risk-benefit assessment of surgery for adult scoliosis: an analysis based on patient age. Spine (Phila Pa 1976). 2011;36:817-24.

9. Puvanesarajah V, Shen FH, Cancienne JM, Novicoff WM, Jain A, Shimer AL, et al. Risk factors for revision surgery following primary adult spinal deformity surgery in patients 65 years and older. J Neurosurg Spine. 2016;25:486-93.

10. Kelly MP, Lenke LG, Bridwell KH, Agarwal R, Godzik ], Koester L. Fate of the adult revision spinal deformity patient: a single institution experience. Spine (Phila Pa 1976). 2013;38:E1196-200.

11. Schwab FJ, Hawkinson N, Lafage V, Smith JS, Hart R, Mundis G, et al. Risk factors for major peri-operative complications in adult spinal deformity surgery: a multi-center review of 953 consecutive patients. Eur Spine J. 2012;21:2603-10.

12. Cho SK, Bridwell KH, Lenke LG, Yi J-S, Pahys JM, Zebala LP, et al. Major complications in revision adult deformity surgery: risk factors and clinical outcomes with 2- to 7-year follow-up. Spine (Phila Pa 1976). 2012;37:489-500.

13. Glassman SD, Dimar JR 2nd, Carreon LY. Revision rate after adult deformity surgery. Spine Deform. 2015;3:199-203.

14. Pichelmann MA, Lenke LG, Bridwell KH, Good CR, O'Leary PT, Sides BA. Revision rates following primary adult spinal deformity surgery: six hundred forty-three consecutive patients followed-up to twentytwo years postoperative. Spine (Phila Pa 1976). 2010;35:219-26.

15. Poorman GW, Zhou PL, Vasquez-Montes D, Horn S, Bortz C, Segreto $\mathrm{F}$, et al. Differences in primary and revision deformity surgeries: following 1,063 primary thoracolumbar adult spinal deformity fusions over time. J spine Surg (Hong Kong). 2018;4:203-10.

16. Kim YJ, Bridwell KH, Lenke LG, Glattes CR, Rhim S, Cheh G. Proximal junctional kyphosis in adult spinal deformity after segmental posterior spinal instrumentation and fusion: minimum five-year follow-up. Spine (Phila Pa 1976). 2008;33:2179-84.

17. Bridwell KH, Lenke LG, Cho SK, Pahys JM, Zebala LP, Dorward IG, et al. Proximal junctional kyphosis in primary adult deformity surgery: evaluation of 20 degrees as a critical angle. Neurosurgery. 2013;72:899-906.

18. Cahill PJ, Wang W, Asghar J, Booker R, Betz RR, Ramsey C, et al. The use of a transition rod may prevent proximal junctional kyphosis in the thoracic spine after scoliosis surgery: a finite element analysis. Spine (Phila Pa 1976). 2012;37:E687-95.

19. Kebaish KM, Martin CT, O'Brien JR, LaMotta IE, Voros GD, Belkoff SM. Use of vertebroplasty to prevent proximal junctional fractures in adult deformity surgery: a biomechanical cadaveric study. Spine J. 2013;13:1897-903.

20. Pehlivanoglu T, Erdag Y, Oltulu I, Akturk UD, Korkmaz E, Yildirim K, et al. Unilateral posterior surgery for severe osteoporotic vertebrae fractures' sequelae in geriatric population: minimum 5-year results of 109 patients. Neurospine. 2021;18:319-27.

21. Albert T], Pinto $M$, Denis $F$. Management of symptomatic lumbar pseudarthrosis with anteroposterior fusion. A functional and radiographic outcome study. Spine (Phila Pa 1976). 2000;25:123-9; discussion 130.

22. Pateder DB, Park Y-S, Kebaish KM, Cascio BM, Buchowski JM, Song $\mathrm{EW}$, et al. Spinal fusion after revision surgery for pseudarthrosis in adult scoliosis. Spine (Phila Pa 1976). 2006;31:E314-9.

23. Etminan M, Girardi FP, Khan SN, Cammisa FPJ. Revision strategies for lumbar pseudarthrosis. Orthop Clin North Am. 2002;33:381-92.

24. Kim Y], Bridwell KH, Lenke LG, Rhim S, Cheh G. Pseudarthrosis in long adult spinal deformity instrumentation and fusion to the sacrum: prevalence and risk factor analysis of 144 cases. Spine (Phila Pa 1976). 2006;31:2329-36.

25. Weistroffer JK, Perra JH, Lonstein JE, Schwender JD, Garvey TA, Transfeldt EE, et al. Complications in long fusions to the sacrum for adult scoliosis: minimum five-year analysis of fifty patients. Spine (Phila Pa 1976). 2008;33:1478-83.

26. Hassanzadeh H, Jain A, El Dafrawy MH, Ain MC, Mesfin A, Skolasky RL, et al. Three-column osteotomies in the treatment of spinal deformity in adult patients 60 years old and older: outcome and complications. Spine (Phila Pa 1976). 2013;38:726-31.

27. Salvi G, Aubin C-E, Le Naveaux F, Wang X, Parent S. Biomechanical analysis of ponte and pedicle subtraction osteotomies for the surgical correction of kyphotic deformities. Eur Spine J. 2016;25:2452-60.

28. Dickson DD, Lenke LG, Bridwell KH, Koester LA. Risk factors for and assessment of symptomatic pseudarthrosis after lumbar pedicle subtraction osteotomy in adult spinal deformity. Spine (Phila Pa 1976). 2014;39:1190-5. 